LETTER TO THE EDITOR

\title{
Short-term secretory regulation of the active form of ghrelin and total ghrelin during an oral glucose tolerance test in patients with anorexia nervosa
}

Yoshikatsu Nakai ${ }^{1}$, Hiroshi Hosoda ${ }^{2,3}$, Kazuko Nin ${ }^{4}$, Chizuru Ooya ${ }^{1}$, Hiromi Hayashi ${ }^{1}$, Takashi Akamizu ${ }^{2}$ and Kenji Kangawa ${ }^{2,3}$

${ }^{1}$ School of Health Sciences, Faculty of Medicine, Kyoto University, Kyoto, Japan, ${ }^{2}$ Translational Research Center, Kyoto University Hospital, Kyoto, 606-8507, Japan, ${ }^{3}$ National Cardiovascular Center Research Institute, Osaka, Japan and ${ }^{4}$ Shiga University of Medical Science, Shiga, Japan

(Correspondence should be addressed to Y Nakai; Email: ynakai@hs.med.kyoto-u.ac.jp)

Ghrelin is an acylated peptide whose octanoyl modification is essential for its biological activities (1). Antibodies used in previous works to measure ghrelin concentrations in human blood do not distinguish between the active form of ghrelin (active ghrelin) and desacyl ghrelin with no biological activities $(2,3)$.
In our previous study, high levels of active ghrelin were present in patients with restricting-type anorexia nervosa (AN-R) and they decreased significantly during an oral glucose tolerance test (OGTT) in these patients as well as in normal controls (4). As a continuation of the previous study and using the same subjects
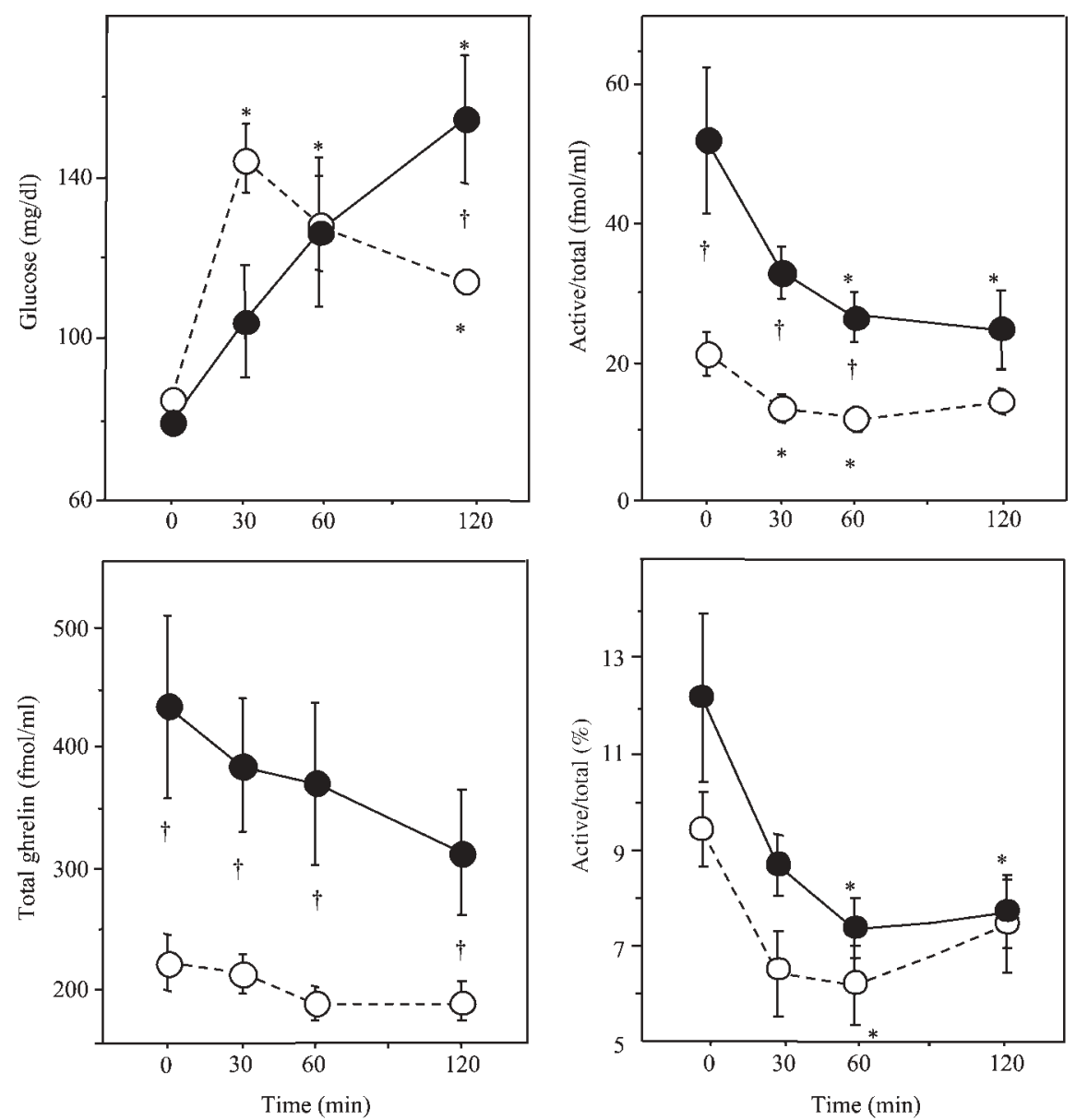

Figure 1 Plasma levels of glucose, total ghrelin and active ghrelin and the ratios of active ghrelin to total ghrelin (active/total) during an OGTT in five patients with AN-R $(\bullet)$ and seven normal controls $(O)$. Data are shown as means \pm S.E.M. ${ }^{*} P<0.01$ vs basal values at 0 min; $† P<0.01$ vs normal controls. 
(4), we studied short-term secretory regulation of active ghrelin as well as total ghrelin during OGTT using two ghrelin-specific radioimmunoassays (5). The details of the study subjects and methods have been described previously $(4,5)$. All results are expressed as means \pm S.E.M. Statistical analysis was performed with the Mann-Whitney $U$ test or the repeated measures ANOVA and subsequently with Dunnett's test. Probabilities of less than 0.05 were considered statistically significant.

Figure 1 shows the plasma levels of glucose, total ghrelin and active ghrelin and the ratios of active ghrelin to total ghrelin during OGTT in patients with AN-R and in controls. Mean basal ratios of active ghrelin to total ghrelin were $12.2 \pm 1.7 \%$ in anorectic patients and $9.4 \pm 0.8 \%$ in controls respectively. These results suggest that desacyl ghrelin predominates over active ghrelin in the circulation in anorectic patients as well as in controls.

Mean levels of plasma total ghrelin did not decrease significantly during OGTT in patients with AN-R and in controls. However, mean levels of plasma active ghrelin decreased significantly during OGTT in anorectic patients and in controls. Furthermore, the mean ratios of active ghrelin to total ghrelin also decreased significantly in these patients and in controls (Fig. 1). These results suggested that active ghrelin disappears from the circulation more rapidly than total ghrelin during OGTT in patients with AN-R and in controls.

Although the plasma levels of both active ghrelin and total ghrelin were significantly higher in anorectic patients compared with normal controls, the difference of the ratios of active ghrelin to total ghrelin was not statistically significant at 0, 30, 60 and $120 \mathrm{~min}$ during OGTT between the two groups (Fig. 1). These results suggest that the short-term secretory regulation of ghrelin during OGTT in patients with AN-R was similar to that in normal controls, despite the high plasma levels of active ghrelin and total ghrelin in these patients compared with normal controls.

\section{References}

1 Kojima M, Hosoda H, Date Y, Nakazato M, Matsuo H \& Kangawa K. Ghrelin is a growth-hormone-releasing acylated peptide from stomach. Nature $1999 \mathbf{4 0 2} 656-660$.

2 Otto B, Cuntz U, Fruehauf E, Wawarta R, Folwaczny C, Riepl RL et al. Weight gain decreases elevated plasma ghrelin concentrations of patients with anorexia nervosa. European Journal of Endocrinology 2001145 R5-R9.

3 Shiiya T, Nakazato M, Mizuta M, Date Y, Mondal MS, Tanaka M et al. Plasma ghrelin levels in lean and obese humans and the effect of glucose on ghrelin secretion. Journal of Clinical Endocrinology and Metabolism 200287 240-244.

4 Nakai Y, Hosoda H, Nin K, Ooya C, Hayashi H, Akamizu T et al. Plasma levels of active form of ghrelin during oral glucose tolerance test in patients with anorexia nervosa. European Journal of Endocrinology 2003149 R1-R3.

5 Hosoda H, Kojima M, Matsuo H \& Kangawa K. Ghrelin and desacyl ghrelin: two major forms of rat ghrelin peptide in gastrointestinal tissue. Biochemical and Biophysical Research Communications 2000279 909-913.

Received 17 February 2004

Accepted 17 February 2004 